\title{
Path Loss Characterization Using Machine Learning Models for GS-to-UAV-Enabled Communication in Smart Farming Scenarios
}

\author{
Sarun Duangsuwan $\mathbb{D}^{1},{ }^{1}$ Phakamon Juengkittikul, ${ }^{2}$ and Myo Myint Maw $^{3}$ \\ ${ }^{1}$ Electrical Engineering, Department of Engineering, Prince of Chumphon Campus, \\ King Mongkut's Institute of Technology Ladkrabang (KMITL), Pathio, Chumphon 86160, Thailand \\ ${ }^{2}$ Electrical Engineering, Faculty of Engineering, King Mongkut's Institute of Technology Ladkrabang (KMITL), \\ Bangkok, Thailand \\ ${ }^{3}$ Department of Computer Engineering and Information Technology, Mandalay Technological University (MTU), \\ Patheingyi Township, Mandalay, Myanmar
}

Correspondence should be addressed to Sarun Duangsuwan; ax_sarun@hotmail.com

Received 11 January 2021; Revised 20 June 2021; Accepted 24 July 2021; Published 3 August 2021

Academic Editor: Lorenzo Luini

Copyright (C) 2021 Sarun Duangsuwan et al. This is an open access article distributed under the Creative Commons Attribution License, which permits unrestricted use, distribution, and reproduction in any medium, provided the original work is properly cited.

\begin{abstract}
The purpose of this paper was to predict the path loss characterization of the ground-to-air (G2A) communication channel between the ground sensor (GS) and unmanned aerial vehicle (UAV) using machine learning (ML) models in smart farming (SF) scenarios. Two ML algorithms such as support vector regression (SVR) and artificial neural network (ANN) were studied to analyze the measured data in different scenarios with Napier and Ruzi grass farms as the measurement locations. The proposed empirical GS-to-UAV two-ray (GUT-R) model and the ML models were compared to characterize path loss prediction models. The performances of the path loss prediction models were evaluated using the statistical error indicators in different measurement locations and UAV trajectories. To obtain the statistical error indicators, the accuracy path loss results of UAV trajectory at $2 \mathrm{~m}$ altitudes showed the SVR model $\left(\mathrm{MAE}=1.252 \mathrm{~dB}, \mathrm{RMSE}=3.067 \mathrm{~dB}\right.$, and $\left.R^{2}=0.972\right)$ and the ANN model $(\mathrm{MAE}=1.150 \mathrm{~dB}$, RMSE $=2.502 \mathrm{~dB}$, and $\left.R^{2}=0.981\right)$ for the Napier scenario. In the Ruzi scenario, the SVR model $(\mathrm{MAE}=1.202 \mathrm{~dB}$, $\mathrm{RMSE}=2.962 \mathrm{~dB}$, and $\left.R^{2}=0.965\right)$ and the ANN model $\left(\mathrm{MAE}=1.146 \mathrm{~dB}, \mathrm{RMSE}=2.507 \mathrm{~dB}\right.$, and $\left.R^{2}=0.983\right)$ were presented. For UAV trajectory at $5 \mathrm{~m}$ altitudes, the SVR model $\left(\mathrm{MAE}=2.125 \mathrm{~dB}, \mathrm{RMSE}=4.782 \mathrm{~dB}\right.$, and $\left.R^{2}=0.933\right)$ and the ANN model $\left(\mathrm{MAE}=2.025 \mathrm{~dB}, \mathrm{RMSE}=4.439 \mathrm{~dB}\right.$, and $\left.R^{2}=0.950\right)$ were resulted in the Napier scenario. In the Ruzi scenario, the SVR model $\left(\mathrm{MAE}=2.112 \mathrm{~dB}, \mathrm{RMSE}=4.682 \mathrm{~dB}\right.$, and $\left.R^{2}=0.935\right)$ and the $\mathrm{ANN}$ model $\left(\mathrm{MAE}=2.016 \mathrm{~dB}, \mathrm{RMSE}=4.407 \mathrm{~dB}\right.$, and $\left.R^{2}=0.954\right)$ were displayed. The proposed ML models using SVR and ANN can optimally predict the path loss characterization in SF scenarios, where the accuracy was $95 \%$ for the SVR and $97 \%$ for the ANN.
\end{abstract}

\section{Introduction}

SF is a global trend to produce new opportunities for agricultural farming. For agriculture 4.0, the solution of SF needs automation systems, robotics, information services, information and communication technologies (ICT), UAVused cases (both fixed- and rotary-wing), artificial intelligence (AI), big data analytics, and the Internet of things (IoT). UAV-based communications are the solution to raise the services in SF because it has rapidly expanded to all areas of agriculture, including pesticide and fertilizer spraying, seed sowing, monitoring, growth assessment, and mapping [1]. UAV applications in SF, such as mapping, spraying, planting, harvesting, irrigation, and crop monitoring, are also existent. For mapping, UAVs can be used to conduct 3dimensional (3D) mapping of agricultural fields, including the surveying area of farmland, soil monitoring, and status of the crop with a high-resolution map [2]. Additionally, the planting application can be carried out by UAV [3]. Such a system is used to distribute seeds and plant nutrients when sowing to provide perfect conditions for plant growth. Moreover, the UAV can identify the areas where water is 
scarce based on image processing and data acquisition for the irrigation system. The use of UAVs equipped with spectral sensors can obtain data for water management and irrigation system [4]. In [5], simultaneous localization and mapping (SLAM) technology in real-time mapping was presented using LiDAR to obtain data for low altitude imaginary. It can recognize the position and identify obstacles when the UAV tasks are performed. To monitor the data acquisition, UAVs are equipped with multifunctional sensors in a mobility network, especially in areas lacking mobile station networks in the field [6].

Focusing on UAV-based communications in SF scenarios, such as air-to-ground (A2G) communication or ground-to-air (G2A) communication, has been described using UAVs to communicate with IoT devices or GS such as soil moisture sensors, humidity sensors, or multienvironmental sensors. To realize reliable communications, an analytical channel model between GS and UAV requires the propagation characteristic under certain SF scenarios [7]. The received signal strength indicator (RSSI) and path loss model are significantly essential parameters for link budget analysis. For the GS-to-UAV communication link, the power signal strength is limited at GS when the signal is transmitted from GS to the receiver on the UAV. Consequently, an adequate coverage zone for GS is limited. In this situation, the communication link will be degraded, particularly when the signal propagates through free space or dense crops. For that reason, the transmission link quality and propagation performance variable must be considered when the multiple GS are deployed in SF. The propagation characteristics of GS-to-UAV are severely impacted by the surrounding environments. Both RSSI and path loss characteristics are the link budget parameters that are employed for critical network design aspects such as the proper level of UAV trajectory and GS locations, coverage prediction, coverage zone, etc. However, the complex SF environment in such networks makes path loss prediction more difficult. Hence, a precise path loss model in the wireless channel must be addressed to predict the propagation characteristics in SF [8]. Likewise, highly accurate models are required to predict the path loss model and guarantee efficiency between GS and UAV communications. Hereby, ML models are the solution for highly accurate prediction models. Moreover, the application of ML models can be used as the new data prediction models in several works [9-15].

To survey the related works of the path loss prediction models, the model of ML-based ANN and RF algorithms was presented in [16]. The performance indices show that the proposed RF model had the best performance than the ANN model. Although the performance of the ANN model could not outperform the RF, the study in [17] shows that it can be optimized for the path loss prediction in a very high frequency (VHF) wireless channel. The use of multilayer perceptron (MLP) was accomplished at nine hidden neurons and hyperbolic tangent sigmoid activation function, and the LM learning algorithm was optimized with the probabilistic parameters such as mean absolute error (MAE), mean square error (MSE), root mean square error (RMSE), standard deviation (SD), and the coefficient of determination or $R$-square $\left(R^{2}\right)$ values. In [18], the ANN algorithm was presented to predict the path loss characterized at $450 \mathrm{MHz}, 850 \mathrm{MHz}, 1800 \mathrm{MHz}$, and $2600 \mathrm{MHz}$ for the heterogeneous network (HetNet), where the results of MAE were close to zero and SD was less than $7 \mathrm{~dB}$. In [19], four ML methods such as backpropagation neural network (BPNN), SVR, RF, and AdaBoost were used to predict path loss models for aircraft cabin scenarios. The results showed that the best performance for the lowest prediction error was achieved by the RF method. Thus, it can be seen that the RF method would be suitable for the optimal path loss of measured data in an indoor environment or small-scale fading scenarios. Additionally, the performance of SVR has similar results to BPNN for outdoor environments or largescale fading scenarios $[20,21]$. Thus, the path loss model using deep learning neural network was proposed to improve the prediction error for $5 \mathrm{G}$ mobile communication [22]. At the $2.6 \mathrm{GHz}$ frequency band, the proposed model with deep neural network (DNN) was studied by comparing the ray-tracing model and the uMa 38.901 model. The results show that the DNN method can reduce the prediction error by $4.7 \mathrm{~dB}$ when it is compared with the ray-tracing model and empirical model for the satellite image application. In [23], the authors presented the multiple linear regression model to predict the path loss model for millimeter-wave (MmWave) urban communication. In [24], the accuracy of path loss prediction was proposed using the DNN method to obtain observation data for the ray-tracing model. Toward this end, ML techniques are capable of assessing the wireless channel. In particular, the survey on ML techniques for UAV-based communications was presented in [25]. Nevertheless, there are a few considerations of ML models for predicting the path loss characterization in SF scenarios.

Using ML techniques in SF to predict the path loss characteristics is performed to the reliable communication between the GS location and UAV trajectory.

The major contributions and novelties of this paper are as follows:

(1) The new channel model called GS-to-UAV communications in SF is presented by investigating RSSI and path loss characteristics.

(2) RSSI and path loss characteristics are predicted using two ML models such as SVR and ANN models. The SVR and ANN models are considered because these algorithms are appropriate to evaluate the data driven in the large-scale fading scenarios. Also, the path loss prediction is a supervised regression problem; thus, it can be solved by SVR and ANN algorithms.

(3) The validation model and results are evaluated with the statistical error indicators such as MAE, RMSE, and $R^{2}$ and compared with the empirical GUT-R path loss model.

The remainder of this paper is organized as follows. The methodology is presented in Section 2 where the measurement setup, the empirical GUT-R model, and the ML models are described. Section 3 presents the results of the 
comparative performance of RSSI-based prediction models and path-loss-based prediction models. Finally, the conclusion is described in Section 4.

\section{Methodology}

The application of the GS and UAV-enabled communication in $\mathrm{SF}$ is described in this section. Due to the limit of radiation energy from the GS, UAV as a mobile base station (MBS) can be equipped with a transceiver module to transmit signals for multiple GS. Figure 1 shows the system model of soil moisture monitoring using multiple GS and a single UAVbased communication for SF. The system model illustrates that multiple GS are installed in the large-scale farm for sensing the soil moisture values. The GS consists of solar cell panels to supply the energy for the capacitive soil moisture sensor and the module microcontroller unit or MCU node. Thus, the communication link between GS and UAV is based on Wi-Fi standard IEEE $802.11 \mathrm{~g}$. The voltage and current of the GS module are used at $3.3 \mathrm{~V}$ and $240 \mathrm{~mA}$, respectively. The benefit of this system model is helping the users monitor soil moisture data as rapidly in the large-scale farm [26].

To evaluate the RSSI and path loss characterization between the GS and UAV of the system model, the measurement setup, GUT-R model, and ML model are described in Sections 2.1, 2.2, and 2.3, respectively.

2.1. Measurement Setup. The geometric 3D model for the measurement setup is considered using the Cartesian coordinate system, as shown in Figure 2. The Cartesian $(x, y, z)$ system represents the position of GS installation and UAV altitude. The UAV altitudes in $z$-axis, $h_{\mathrm{UAV}}$, were set at $2 \mathrm{~m}$ and $5 \mathrm{~m}$. Additionally, the height of GS, $h_{g}$, was set at $1.5 \mathrm{~m}$, while the horizontal distance, $d_{c}$, was set from the GS point until $20 \mathrm{~m}$. In the measurement, the UAV was equipped with a Wi-Fi module transceiver to communicate with the GS.

In order to measure the RSSI and path loss characteristics, we consider a single GS test kit position at the Cartesian $\left(x_{3}, y_{3}, z\right)$ or Napier33 position as well as the GS position at the Cartesian $\left(x_{1}, y_{3}, z\right)$ or Ruzi13 position. In this experiment, the GS test kit and UAV were connected using $\mathrm{Wi}-\mathrm{Fi} 2.4 \mathrm{GHz}$ frequency wireless links, and the measurement data of RSSI can record through the Internet by the application program interface (API) software as shown in Figure 3. After that, the path loss values can be calculated in the offline postprocessing. Figure 4 shows the top view of the measurement location and side view of the measurement setup in the Napier scenario. The dimension of the Napier scenario was $25 \mathrm{~m}$ wide and $45 \mathrm{~m}$ long. Figure 5 shows the top view of the measurement location and the side view of the Ruzi scenario, while the dimension of the Ruzi scenario was $30 \mathrm{~m}$ wide and $50 \mathrm{~m}$ long. Table 1 shows the measurement setup parameters. In this work, the measurement locations were experimentally performed at the Tropical Animal Research Institute, Ramkhamhaeng University, Thailand.
2.2. GUT-R Model. The propagation characteristics between GS and UAV can be investigated using the empirical GUT-R model. Figure 6 shows the geometrics of the GUT-R model where the total received $E$-field at the UAV is $E_{U}\left(d_{c}\right)$ and then the result of the direct line-of-sight (LOS) component, $E\left(d^{\prime}\right)$, and the ground reflected component, $E_{r}\left(d^{\prime \prime}\right)$. The expression is as follows:

$$
E_{U}\left(d_{c}\right)=E\left(d^{\prime}\right)+E_{r}\left(d^{\prime \prime}\right) .
$$

The traveling two waves are noted at UAV: the direct wave that travels distance, $d^{\prime}$, and the reflected wave that travels distance, $d^{\prime \prime}$. The E-field due to the LOS at the UAV can be expressed as follows:

$$
E\left(d^{\prime}\right)=\frac{E_{0} d_{0}}{d^{\prime}} \cos \left(2 \pi f_{c}\left(t-\frac{d^{\prime}}{c}\right)\right),
$$

and the $E$-field for the ground reflected wave, which has a propagation distance of $d^{\prime \prime}$, can be expressed as follows:

$$
E_{r}\left(d^{\prime \prime}\right)=\Gamma \frac{E_{0} d_{0}}{d^{\prime \prime}} \cos \left(2 \pi f_{c}\left(t-\frac{d^{\prime \prime}}{c}\right)\right),
$$

where $f_{c}$ is the carrier frequency and $E_{0}$ is the free-space $E$-field at a reference distance $d_{0}$ from the GS; then, $d>d_{0}$. $\Gamma$ denotes the reflection coefficient for ground.

The $E_{U}\left(d_{c}\right)$ can be expressed as the sum of equations (2) and (3) as follows:

$$
\begin{aligned}
E_{U}\left(d_{c}\right)= & \frac{E_{0} d_{0}}{d^{\prime}} \cos \left(2 \pi f_{c}\left(t-\frac{d^{\prime}}{c}\right)\right) \\
& +(-1) \frac{E_{0} d_{0}}{d^{\prime \prime}} \cos \left(2 \pi f_{c}\left(t-\frac{d^{\prime \prime}}{c}\right)\right),
\end{aligned}
$$

where $\Gamma=-1$ denotes the perfect ground reflection component.

Considering the UAV altitude $h_{\mathrm{UAV}}$ and GS height $h_{g}$, the path difference between the LOS and ground reflection path can be expressed as follows:

$$
\Delta d=\frac{2 h_{\mathrm{UAV}} h_{g}}{d_{c}} .
$$

The phase difference $\Delta \theta$ between the two-ray model components and the time delay $\tau_{d_{c}}$ between the arrival of the two components can be given as follows:

$$
\begin{gathered}
\Delta \theta=\frac{2 \pi \Delta d_{c}}{\lambda}, \\
\tau_{d_{c}}=\frac{\Delta \theta}{2 \pi f_{c}} .
\end{gathered}
$$

When the distance $d_{c}$ is larger, the reflection distance $d^{\prime \prime}$ is very small, and the amplitudes of $E\left(d^{\prime}\right)$ and $E_{r}\left(d^{\prime \prime}\right)$ are identical and differ only in phase that can be expressed as follows:

$$
\left|\frac{E_{0} d_{0}}{d}\right| \approx\left|\frac{E_{0} d_{0}}{d^{\prime}}\right| \approx\left|\frac{E_{0} d_{0}}{d^{\prime \prime}}\right|
$$




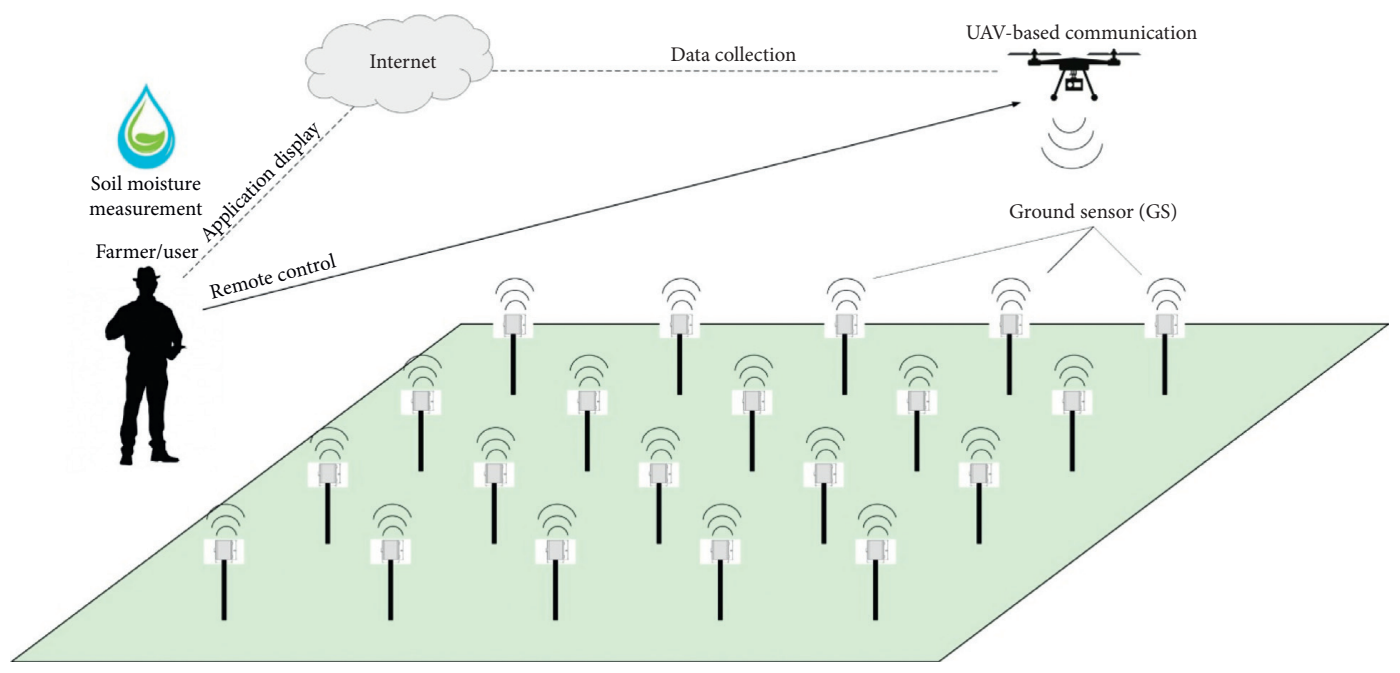

FIgURE 1: The system model for GS-to-UAV-enabled communications in SF.

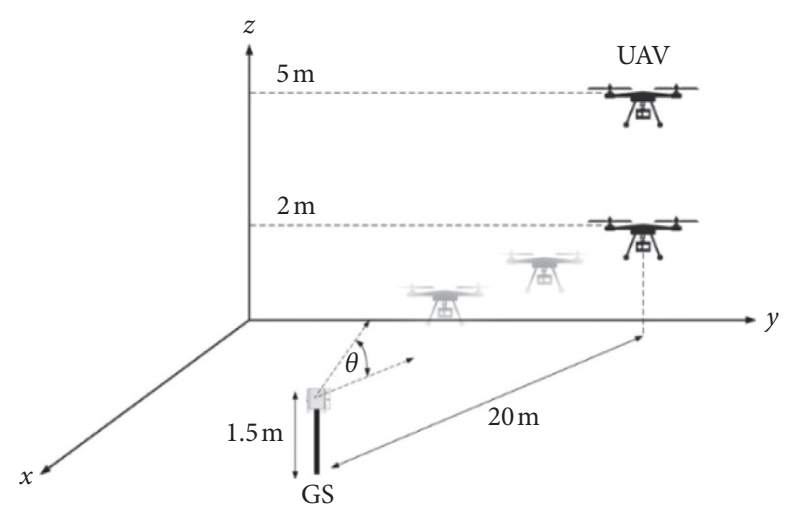

FIgURE 2: The geometric 3D model for measurement setup in SF.

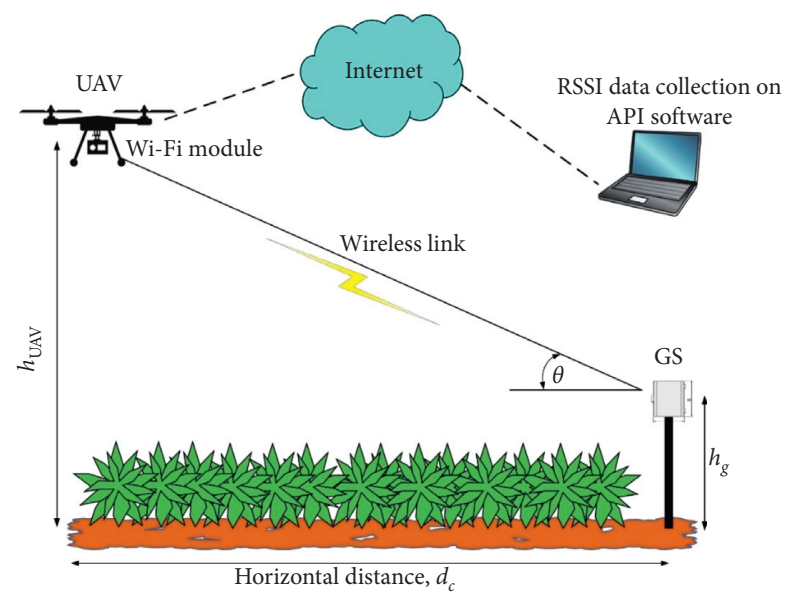

FIgURE 3: The measurement model of GS-to-UAV communication for RSSI data collection in SF. 

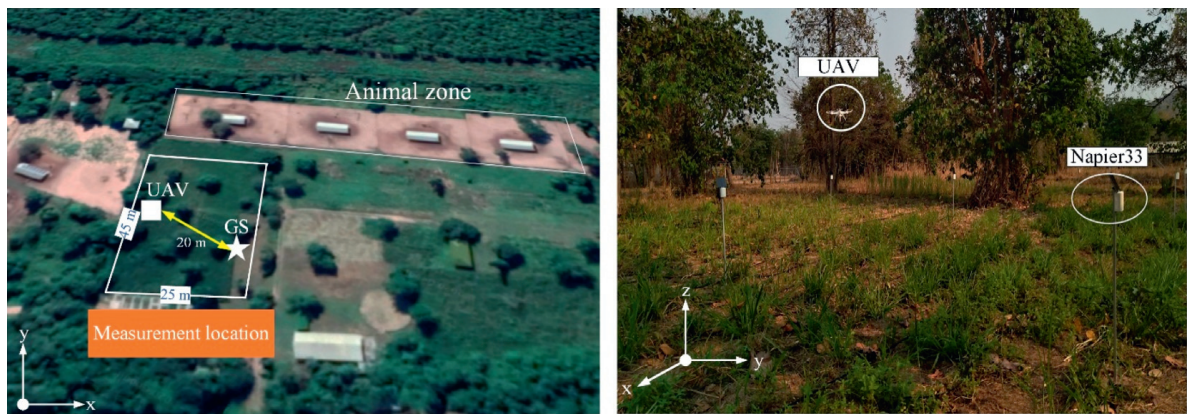

Figure 4: Top view of the measurement location and side view of the example test: UAV trajectory at $2 \mathrm{~m}$ altitudes in the Napier farm.
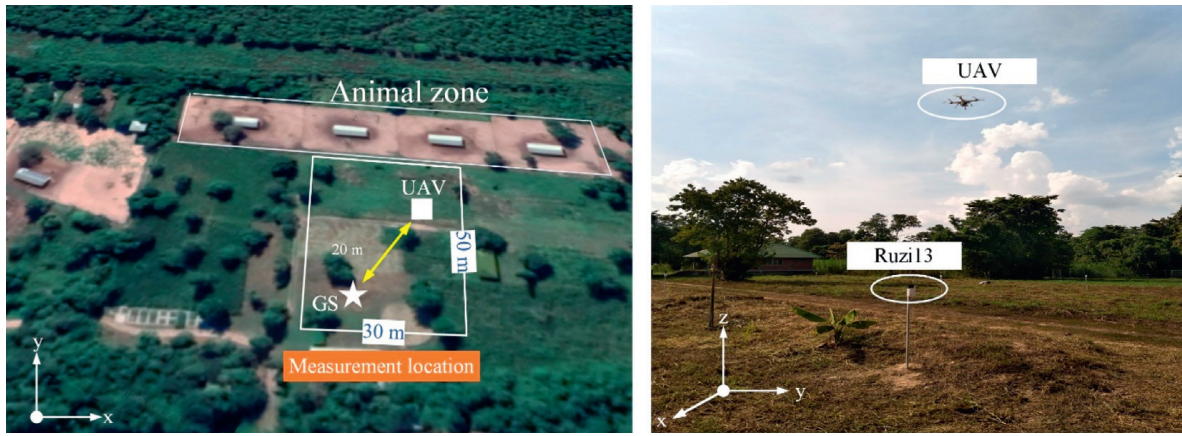

FIgURE 5: Top view of the measurement location and side view of the example test: UAV trajectory at $5 \mathrm{~m}$ altitudes in the Ruzi farm.

TABle 1: Measurement setup parameters.

\begin{tabular}{lc}
\hline Parameters & Values \\
\hline Carrier frequency & $2.4 \mathrm{GHz}$ \\
GS antenna gain (IoT module) & $3.0 \mathrm{dBi}$ \\
UAV antenna gain (Wi-Fi module) & $5.1 \mathrm{dBi}$ \\
Transmitter power (Wi-Fi module) & $20 \mathrm{dBm}$ \\
Horizontal distance & $20 \mathrm{~m}$ \\
UAV altitudes & $2 \mathrm{~m}$ and $5 \mathrm{~m}$ \\
GS height & $1.5 \mathrm{~m}$ \\
\hline
\end{tabular}

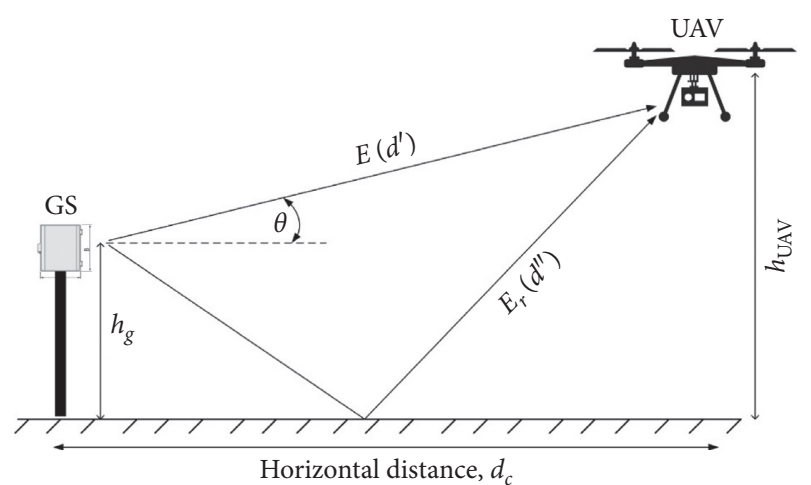

FIGURE 6: The geometrics for the empirical GUT-R model.

The total for the received $E$-field, $E_{U}\left(d_{c}\right)$, in the case of long distance, $d_{c}$, can be expressed as follows:

$$
\begin{aligned}
E_{U}\left(d_{c}\right) & =\frac{E_{0} d_{0}}{d^{\prime}} \cos \left(\omega\left(\frac{d^{\prime \prime}-d^{\prime}}{c}\right)\right)-\frac{E_{0} d_{0}}{d^{\prime \prime}} \cos \Delta \theta \\
\left|E_{U}\left(d_{c}\right)\right| & =\sqrt{\left(\frac{E_{0} d_{0}}{d_{c}}\right)^{2} \cos (\Delta \theta-1)^{2}+\left(\frac{E_{0} d_{0}}{d_{c}}\right)^{2} \sin ^{2} \Delta \theta}
\end{aligned}
$$

In terms of the power received, $P_{\mathrm{UAV}}\left(d_{c}\right)$, it is related to the square of the $E_{U}\left(d_{c}\right) E$-field. The received power at the horizontal distance, $d_{c}$, from the GS for the two-ray model can be expressed as follows:

$$
P_{\mathrm{UAV}}\left(d_{c}\right)=P_{g} G_{g} G_{u}\left(\frac{h_{g}^{2} h_{\mathrm{UAV}}^{2}}{d_{c}^{4}}\right),
$$

where $G_{g}$ and $G_{u}$ represent the antenna gain of GS and the antenna gain of the receiver at the UAV, respectively. $P_{g}$ is the power transmitted from the GS. In equation (11), we note that at large distances, $d_{c} \gg \sqrt{h_{g} h_{\mathrm{UAV}}}$, the received power falls off with the distance raised that is more rapid path loss in free space. At the large values of $d_{c}$, the path loss for the GUT- $R$ model can be expressed in $\mathrm{dB}$ as follows:

$$
\begin{aligned}
P_{L}^{\mathrm{GUT}-\mathrm{R}}(\mathrm{dB})= & 40 \log d_{c}-\left(10 \log G_{g}+10 \log G_{u}\right. \\
& \left.+20 \log h_{g}+20 \log h_{\mathrm{UAV}}\right) .
\end{aligned}
$$

At short distances, $d_{c}$, it can be obtained using equation (4) to compute the total $E_{U}\left(d_{c}\right) E$-field. When the 
substitution in equation (6) is evaluated for $\Delta \theta=\pi / 2$, then $d_{c}=4 h_{g} h_{\mathrm{UAV}} / \lambda$ has appeared.

2.3. Machine Learning Model. The procedure of ML-based RSSI and path loss prediction model is shown in Figure 7. In this section, the data sets are considered as RSSI and path loss from the measured data collection that is separated as the training data set $80 \%$ and test data set $20 \%$, respectively. For such procedure of ML-based prediction model, four sections including the preparation of training and test sets, model selection of SVR and ANN algorithms, model training, and the evaluation by calculating the statistical error indicators such as MAE, RMSE, and $R^{2}$ are performed to build the validation model [27-29]. After the validation model has been built, RSSI and path loss measured data can be generated with new data inputs to predict the output observation using the prediction models.

The model selection of SVR and ANN algorithms is performed in Algorithms 1 and 2.

In thenext step, the evaluated data by the statistical error indicators such as MAE, RMSE, and $R^{2}$ for RSSI and path loss are expressed as follows:

$$
\begin{aligned}
& \text { MAE }= \frac{1}{N} \sum_{i=1}^{N}\left|x_{i, \text { Test }}-x_{\text {Predict }}^{\text {SVR,ANN }}\right|, \\
& \frac{1}{N} \sum_{i=1}^{N}\left|y_{i, \text { Test }}-y_{\text {Predict }}^{\text {SVR,ANN }}\right|, \\
& \text { RMSE }= \sqrt{\left[\frac{1}{N} \sum_{i=1}^{N}\left[x_{i, \text { Test }}-x_{\text {Predict }}^{\text {SVR,ANN }}\right]^{2},\right.} \\
& R^{2}=1-\frac{\sum_{i=1}^{N}\left[x_{i, \text { Test }}-x_{\text {Predict }}^{\text {SVR,ANN }}\right]}{\sum_{i=1}^{N}\left[x_{i, \text { Test }}-\bar{x}_{\text {Predict }}^{\text {SVR,ANN }}\right]}, \\
& 1-\frac{\sum_{i=1}^{N}\left[y_{i, \text { Test }}-y_{\text {Predict }}^{\text {SVR,ANN }}\right]}{\sum_{i=1}^{N}\left[y_{i, \text { Test }}-\bar{y}_{\text {Predict }}^{\text {SVR,ANN }}\right]},
\end{aligned}
$$

where $N=50$ represents the total number of test data sets and $\mathbf{x}_{i, \text { est }}$ and $\mathbf{y}_{i, \text { Test }}$ are the test data sets. The $\bar{x}_{\text {Predict }}^{\mathrm{SVRN}}$ and $\bar{y}_{\text {Predict }}^{\mathrm{SVR}, \mathrm{ANN}}$ are the average of the predicted value.

\section{Evaluation}

In this section, the results of RSSI and path loss characterization of the GUT-R model, the measurement data, the proposed SVR model, and the proposed ANN model are shown. In Section 3.1, the comparative performance results of RSSI-based prediction models are described. The comparative performance of path loss-based prediction models is shown in Section 3.2, and the discussion is described in Section 3.3.
3.1. RSSI-Based Prediction Models. In Figure 8, the RSSI level is powered at $10 \mathrm{dBm}$ to $-32 \mathrm{dBm}$ for UAV trajectory at $2 \mathrm{~m}$ altitudes in the Napier scenario. The horizontal distance between UAV and GS (Napier33 position) is separated from $0 \mathrm{~m}$ to $20 \mathrm{~m}$. It can be observed that the measured data is related to the GUT-R model that depends on free-space loss. Besides, the validation models from the SVR and ANN model curves can predict the RSSI measured data where the statistical errors of the ANN model are smaller than the SVR model as shown in Table 2. To consider the propagation effect of this scenario, we observe that there is some power fluctuated from ground reflection at $3 \mathrm{~m}$ to $5 \mathrm{~m}$ distance, which is reflected into the UAV. Thus, this position may fluctuate the power more than others. As a result of the prediction model, it is clear that the MAE of the SVR model is $1.636 \mathrm{dBm}$ and $1.596 \mathrm{dBm}$ for the ANN model. While the RMSEs of the SVR and ANN models are $3.336 \mathrm{dBm}$ and $3.196 \mathrm{dBm}$, respectively. The $R^{2}$ of the SVR and ANN models are 0.957 and 0.964 , respectively. As a result, it is clear that the ANN model outperforms the SVR model slightly because the predictive curve of ANN model can approach the measured data better than the SVR curve.

In Figure 9, the measured data of RSSI level is $5 \mathrm{dBm}$ to $-28 \mathrm{dBm}$ in the Ruzi scenario. We observe that the effect from ground reflection occurs at a $2 \mathrm{~m}$ distance. To perform the statistical errors, it can be seen that the MAE of the SVR model is $1.121 \mathrm{dBm}$ and $1.112 \mathrm{dBm}$ for the ANN model. The RMSEs of the SVR and ANN models are $1.870 \mathrm{dBm}$ and $1.628 \mathrm{dBm}$, respectively. The $R^{2}$ of the SVR and ANN models are 0.974 and 0.981 , respectively, as shown in Table 2. As a result, the performance of both the SVR and ANN models can absolutely predict the measured data in this scenario.

For UAV trajectory at $5 \mathrm{~m}$ altitudes, the GUT-R model curve is $2 \mathrm{dBm}$ to $-58 \mathrm{dBm}$, while the measured data is $2 \mathrm{dBm}$ to $-70 \mathrm{dBm}$ as shown in Figures 10 and 11, respectively. It can be seen that the measured data is independent of the ground reflection because of the large distance, $d_{c}$. To perform the statistical error indicators, the MAE of the SVR model is $1.785 \mathrm{dBm}$ and $1.698 \mathrm{dBm}$ for the ANN model. The RMSEs of the SVR and ANN models are $3.718 \mathrm{dBm}$ and $3.219 \mathrm{dBm}$, respectively. The $R^{2}$ of the SVR and ANN models are 0.979 and 0.988 , respectively. Similarly, in Figure 10, the MAE of the SVR model is $1.734 \mathrm{dBm}$ and $1.456 \mathrm{dBm}$ for ANN model. The RMSEs of the SVR and ANN models are $3.266 \mathrm{dBm}$ and $2.743 \mathrm{dBm}$, respectively. The $R^{2}$ of the SVR and ANN models are 0.977 and 0.983, respectively. The statistical error indicators of RSSI when UAV trajectory is at $5 \mathrm{~m}$ altitudes are shown in Table 3.

3.2. Path Loss-Based Prediction Models. Figure 12 shows the path loss comparison of the GUT-R model, the measured data, and the prediction models when the UAV trajectory is at $2 \mathrm{~m}$ altitudes in the Napier scenario. The path loss-based GUT-R model ranges from $-24 \mathrm{~dB}$ to $38 \mathrm{~dB}$ in this scenario; however, it can be seen that the measured data is varied from $-20 \mathrm{~dB}$ to $40 \mathrm{~dB}$. The MAE between the GUT-R model and the measured data is $12.214 \mathrm{~dB}$ and $14.334 \mathrm{~dB}$ of RMSE, respectively, as shown in Table 4. Additionally, we can show 


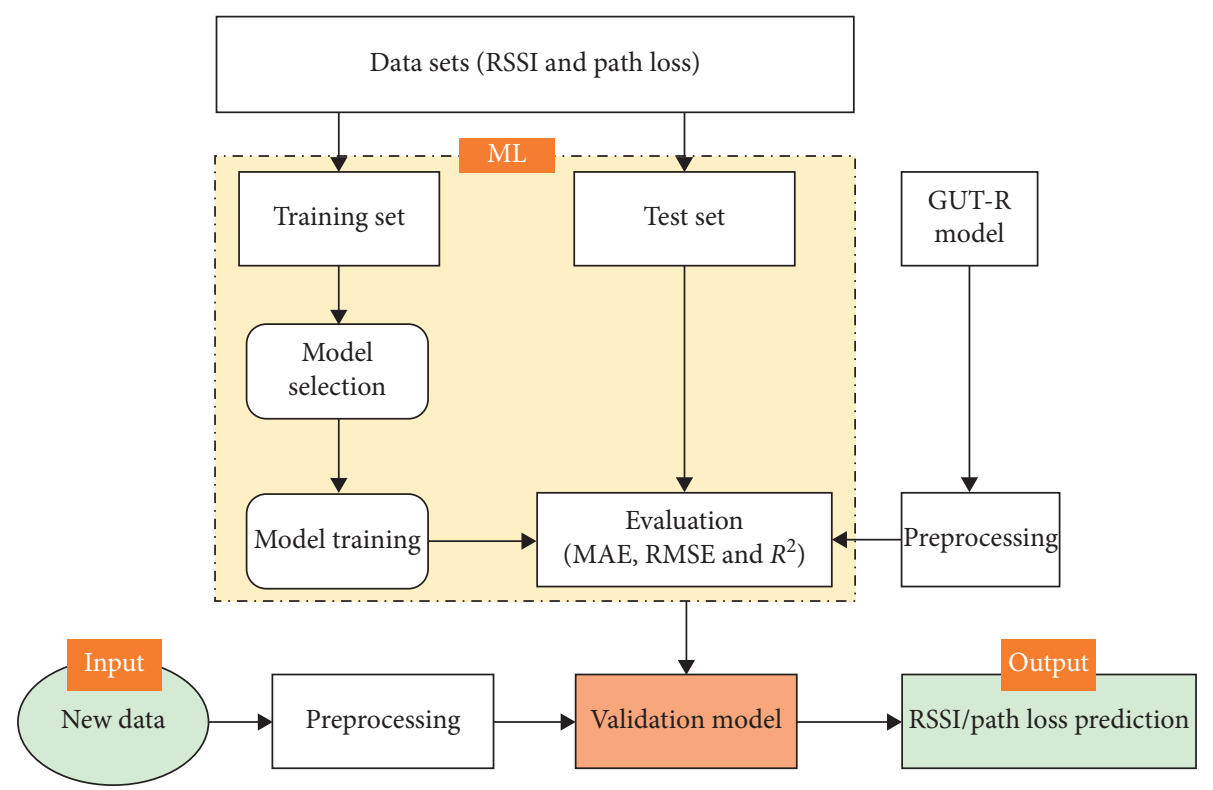

Figure 7: Procedure of ML-based RSSI and path loss prediction model.

Hyperparameter setting:

(1) Let the $\mathbf{x}=\left[r_{i}, d_{c}\right]$ be the RSSI training data sets and $\mathbf{y}=\left[\mathrm{PL}_{i}, d_{c}\right]$ be the path loss training data set. The data set was separated as $80 \%$ for the training set and $20 \%$ for the test set.

(2) Let the function $f(\phi(\mathbf{x}))$ and $f(\phi(\mathbf{y}))$ be the basis function for RSSI and path loss of the hyperparameter setting, respectively. The hyperplane in the feature space is $f(\phi(\mathbf{x}))=\sum_{i=1}^{N}\left(m \cdot x_{i}+b\right)$ and $f(\phi(\mathbf{y}))=\sum_{i=1}^{N}\left(m \cdot y_{i}+b\right)$, where $m$ and $b$ denote the slope and intercept of the regression model.

Model training:

(3) The predicted value is $x_{\text {Predict }}^{\mathrm{SVR}}=\min (1 / 2)\|f(\phi(x))\|^{2}+C \sum_{i=1}^{N}\left(x_{i}+\bar{x}_{i}\right)$ and $\mathbf{y}_{\text {Predict }}^{\mathrm{SVR}}=\min (1 / 2)\|f(\phi(\mathbf{y}))\|^{2}+C \sum_{i=1}^{N}\left(y_{i}+\bar{y}_{i}\right)$ for RSSI and path loss, respectively, where the value $C>0$ controls the iteration between the test and training sets, $\bar{x}_{i}$ and $\bar{y}_{i}$ represent the average mean of RSSI and path loss test set, and $\|\bullet\|$ is the Euclidean norm.

Algorithm 1: SVR.

Hyperparameter setting:

(1) Let $\mathbf{x}=\left[r_{i}, d_{c}\right]$ for RSSI training data sets and $\mathbf{y}=\left[\mathrm{PL}_{i}, d_{c}\right]$ for the path loss training data set. The data set was separated as $80 \%$ for the training set and $20 \%$ for the test set.

(2) Let two input layers $\left[r_{i}, d_{c}\right]$ for RSSI, where $r_{i}$ is the received power level and $d_{c}$ is horizontal distance between GS and UAV. For path loss, $\left[\mathrm{PL}_{i}, d_{c}\right]$ denotes two input layers, where $\mathrm{PL}_{i}$ is the path loss level.

(3) One hidden layer and one output layer using RBF-based neural network (NN) were set, where the number of neurons $k=4$.

(4) The model of ANN is expressed by $\mathbf{x}=f \sum_{j=1}^{k} \mathbf{w}_{j}\left(f \sum_{i=1}^{N} \mathbf{w}_{1 j} \mathbf{x}_{i}\right)$ for RSSI and $\mathbf{y}=f \sum_{j=1}^{k} \mathbf{w}_{j}\left(f \sum_{i=1}^{N} \mathbf{w}_{2 j} \mathbf{y}_{i}\right)$ for path loss, where $\mathbf{w}_{1 j}$ and $\mathbf{w}_{2 j}$ represent the synaptic weights between the two input layers and a hidden layer for input RSSI training set and input path loss training set and $\mathbf{w}_{j}$ denotes the connection weight between the neurons of the hidden and output layers. Model training:

(5) The predicted value of RSSI is $\mathbf{x}_{\mathrm{Predict}}^{\mathrm{ANN}}=\sum_{j=1}^{k} \mathbf{w}_{1 j} \exp \left|-\left(\left\|\mathbf{x}_{i}-c_{j}\right\| / 2 \sigma_{j}^{2}\right)\right|$, and the model of path loss is $\mathbf{y}_{\text {Predict }}^{\text {ANN }}=\sum_{j=1}^{k} \mathbf{w}_{2 j} \exp \left|-\left(\left\|\mathbf{y}_{i}-c_{j}\right\| / 2 \sigma_{j}^{2}\right)\right|$, where $c_{j}$ and $\sigma_{j}^{2}$ are the mean and standard deviation of a Gaussian function, respectively.

Algorithm 2: ANN.

the performance results of the SVR and ANN models that are used to train the training set of the measured data. It can be seen that the performance MAE of the SVR model is 1.252 and 1.150 for the ANN model. The performance RMSEs of the SVR and ANN models are 3.067 and 2.502. $R^{2}$ of the SVR and ANN models are 0.972 and 0.981 , respectively. Although the prediction errors of both the SVR and ANN models are slightly different, the ANN-based prediction model can better predict the path loss precisely in the Napier scenario.

The comparison of path loss characteristics in the Ruzi scenario for the UAV trajectory at $2 \mathrm{~m}$ altitudes is shown in Figure 13. We observe that the measured data increases at 


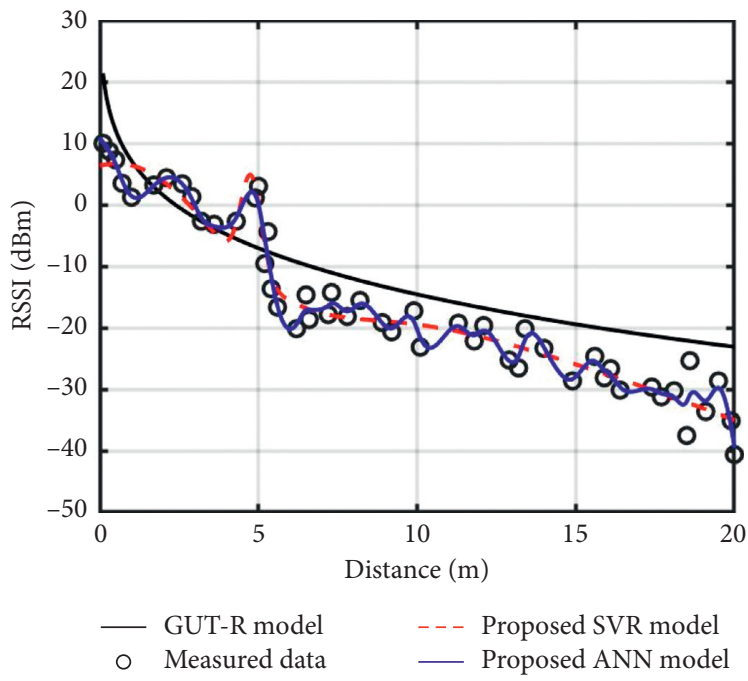

FIgURE 8: RSSI between UAV trajectory at $2 \mathrm{~m}$ altitudes and GS (Napier33) in the Napier scenario.

TABLE 2: RSSI statistical error indicators when UAV trajectory is at $2 \mathrm{~m}$ altitudes.

\begin{tabular}{|c|c|c|c|c|c|c|}
\hline \multirow{2}{*}{ Models } & \multicolumn{3}{|c|}{ Napier scenario } & \multicolumn{3}{|c|}{ Ruzi scenario } \\
\hline & MAE & RMSE & $R^{2}$ & MAE & RMSE & $R^{2}$ \\
\hline GUT-R & 10.590 & 12.290 & 0.334 & 10.246 & 12.539 & 0.289 \\
\hline SVR & 1.636 & 3.336 & 0.957 & 1.121 & 1.870 & 0.974 \\
\hline ANN & 1.596 & 3.196 & 0.964 & 1.112 & 1.628 & 0.981 \\
\hline
\end{tabular}

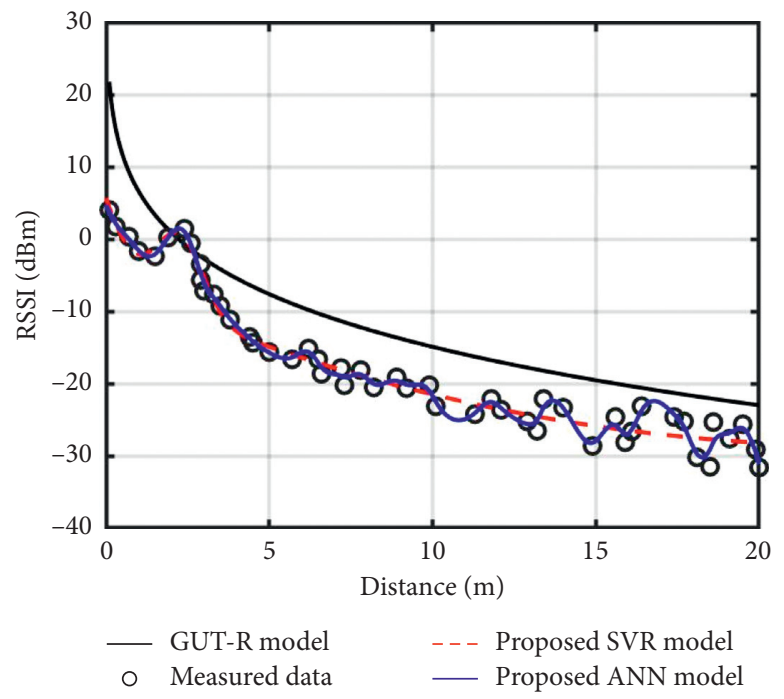

FIgURE 9: RSSI between UAV trajectory at $2 \mathrm{~m}$ altitudes and GS (Ruzi13) in the Ruzi scenario.

$10 \mathrm{~m}$ to $20 \mathrm{~m}$ of the separation distance, and the path loss ranges from $25 \mathrm{~dB}$ to $32 \mathrm{~dB}$. This phenomenon may occur from the scattering surrounding environments. All path loss statistical error indicators are shown in Table 4 for the Ruzi scenario when UAV trajectory is at $2 \mathrm{~m}$ altitudes.

When the UAV trajectory is at $5 \mathrm{~m}$ altitudes, the path loss-based GUT-R model varies from $-22 \mathrm{~dB}$ to $23 \mathrm{~dB}$ because the influence of ground reflection ray in terms of $E_{r}\left(d^{\prime \prime}\right)$ is decreased as shown in Figure 14. Obviously, the prediction model using the ANN model in the blue curve is from $0 \mathrm{~dB}$ to $66 \mathrm{~dB}$. According to the comparison result between the SVR and ANN models, the ANN model can predict the measured data better than the SVR model. Likewise, the RMSE values for the SVR and ANN models are 


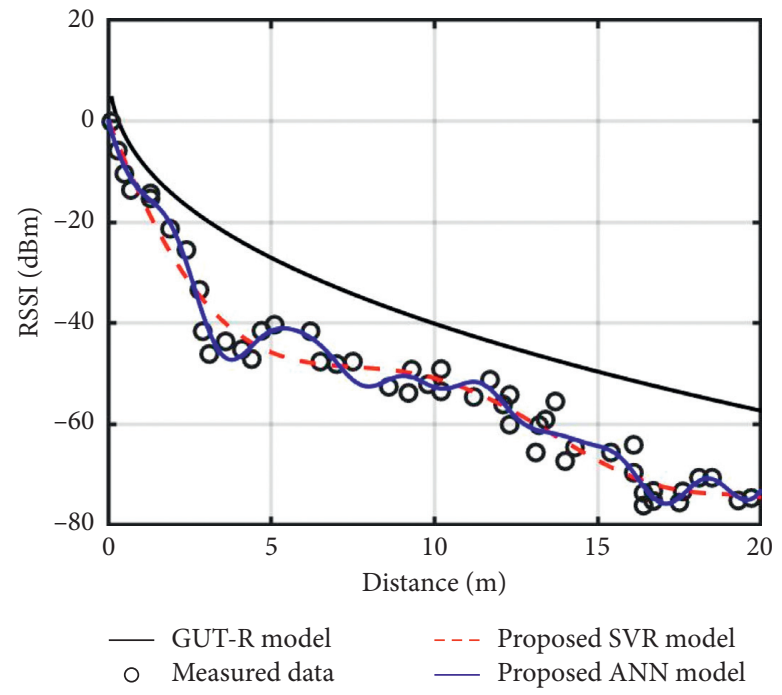

FIgURE 10: RSSI between UAV trajectory at $5 \mathrm{~m}$ altitudes and GS (Napier33) in the Napier scenario.

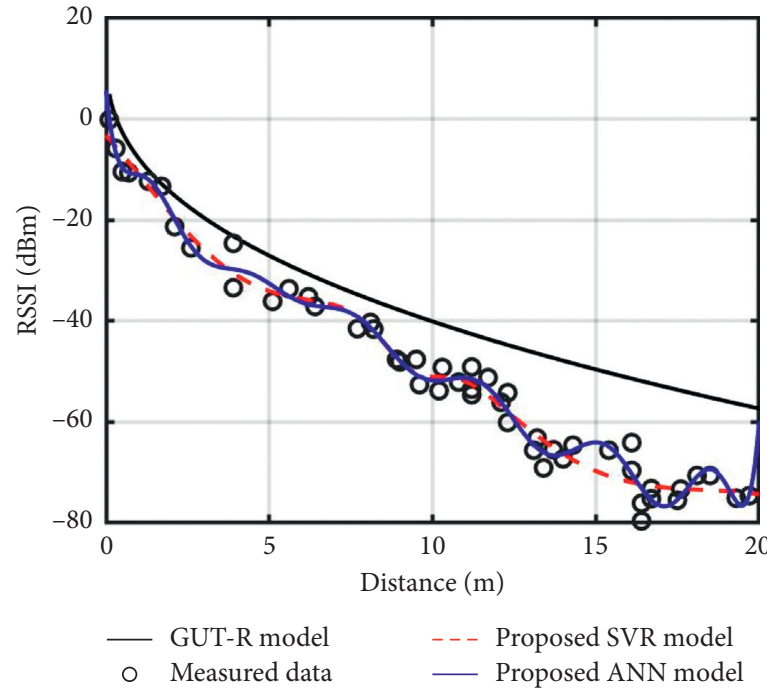

FIGURE 11: RSSI between UAV trajectory at $5 \mathrm{~m}$ altitudes and GS (Ruzi13) in the Ruzi scenario.

TABLE 3: RSSI statistical error indicators when UAV trajectory is at $5 \mathrm{~m}$ altitudes.

\begin{tabular}{lccccr}
\hline \multirow{2}{*}{ Models } & \multicolumn{2}{c}{ Napier scenario } & \multicolumn{3}{c}{ Ruzi scenario } \\
& MAE & RMSE & $R^{2}$ & MAE & RMSE \\
\hline GUT-R & 18.235 & 20.092 & 0.215 & 18.432 & 20.238. \\
SVR & 1.785 & 3.718 & 0.979 & 1.734 & 3.266 \\
ANN & 1.698 & 3.219 & 0.986 & 1.456 & 2.743 \\
\hline
\end{tabular}

better than the GUT-R model when the accuracy of $R^{2}$ is 0.267 for the GUT-R model, 0.933 for the SVR model, and 0.950 for the ANN model as shown in Table 5 .

In Figure 15, the comparison of the measured data and GUT-R model for the Ruzi scenario shows that the RMSE is high up to $32.523 \mathrm{~dB}$ and the accuracy of $R^{2}$ is 0.121 . On the other hand, the prediction errors of the SVR and ANN models are better than the GUT-R model, where the RMSE is
$4.682 \mathrm{~dB}$ for the SVR model and $4.407 \mathrm{~dB}$ for the ANN model.

3.3. Discussion. The result of the path loss characteristic using the empirical GUT-R model depends on parameters such as the antenna gain, GS height, the UAV height, and the horizontal separation distance. Meanwhile, the actual path 


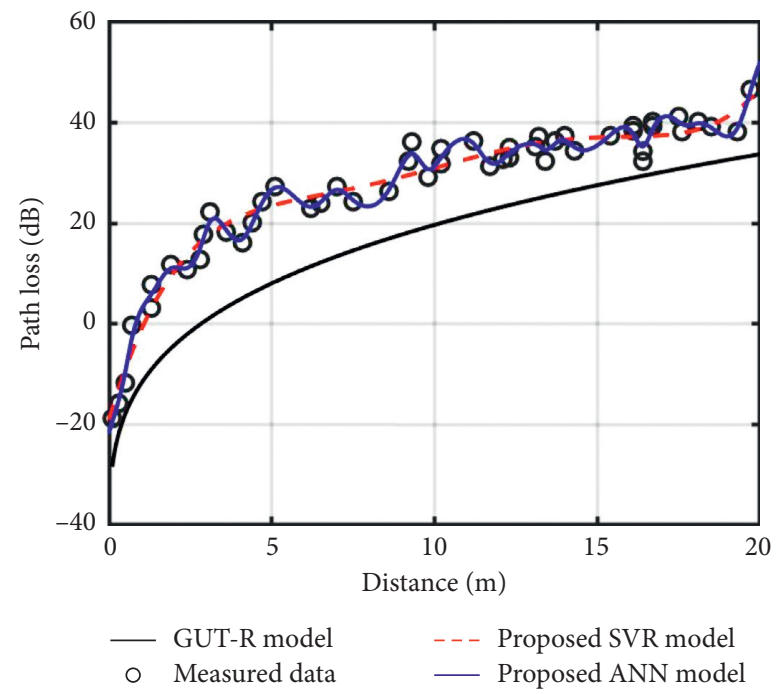

FIgURE 12: Path loss between UAV trajectory at $2 \mathrm{~m}$ altitudes and GS (Napier33) in the Napier scenario.

TABle 4: Path loss statistical error indicators when UAV trajectory is at $2 \mathrm{~m}$ altitudes.

\begin{tabular}{lcccrr}
\hline \multirow{2}{*}{ Models } & \multicolumn{3}{c}{ Napier scenario } & \multicolumn{3}{c}{ Ruzi scenario } \\
& MAE & RMSE & $R^{2}$ & MAE & $R^{2}$ \\
\hline GUT-R & 12.214 & 14.334 & 0.367 & 12.142 & 14.243 \\
SVR & 1.252 & 3.067 & 0.972 & 1.202 & 2.962 \\
ANN & 1.150 & 2.502 & 0.981 & 1.146 & 0.921 \\
\hline
\end{tabular}

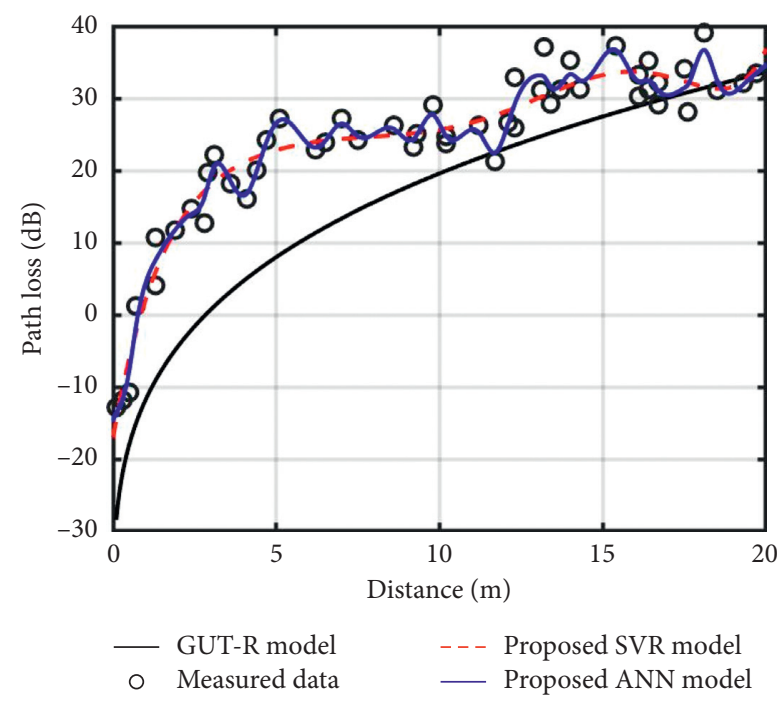

Figure 13: Path loss between UAV trajectory at $2 \mathrm{~m}$ altitudes and GS (Ruzi13) in the Ruzi scenario.

loss measured data is greater than the GUT-R model because the measured data might increase in terms of insertion loss and mismatch polarization loss between GS and UAV antennas. Thus, the consideration of mismatch polarization loss for GS and UAV communications is crucial.

The performance of the ANN model provides better than the SVR model slightly in both the Napier and Ruzi farms of this work. To discuss the performance of the SVR model, the number of $C$ control of the iterative data processing model was done at 230 iterations using MATLAB simulation. To minimize the prediction error between the training and test sets, SVR adopts an insensitive loss function of the predicted value output $\mathbf{x}_{\text {Predict }}^{\text {SVR }}$ and $\mathbf{y}_{\text {Predict }}^{\text {SVR }}$, respectively. The loss function is influenced by a priori information about the noise distribution affecting the data sets. To this end, the $R^{2}$ of the SVR model is 0.951 , or the performance percentage is 95\%. To discuss the ANN model, we found that two input layers as $\left[\mathrm{PL}_{i}, d_{c}\right]$, one hidden layer where the best number 


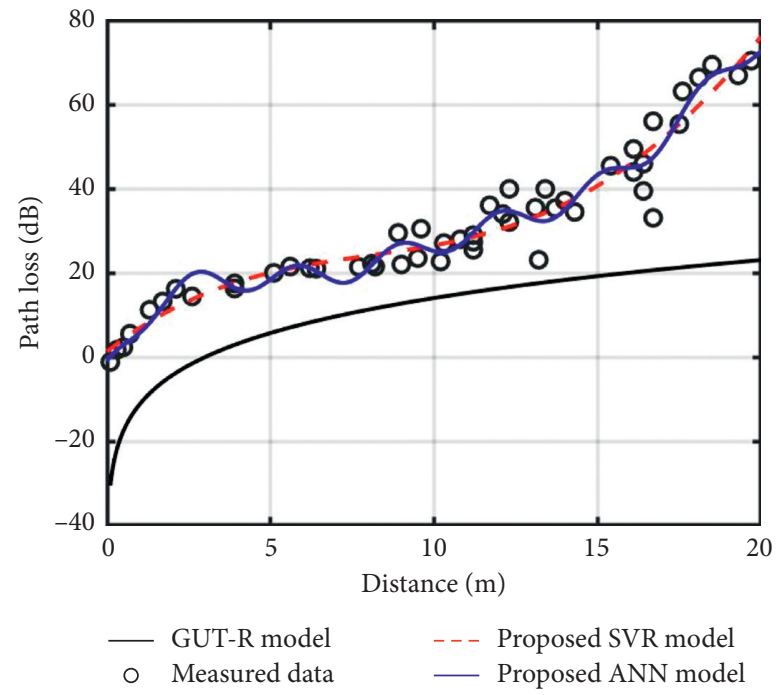

FIgURE 14: Path loss between UAV trajectory at $5 \mathrm{~m}$ altitudes and GS (Napier33) in the Napier scenario.

TABle 5: Path loss statistical error indicators when UAV trajectory is at $5 \mathrm{~m}$ altitudes.

\begin{tabular}{lcccrr}
\hline \multirow{2}{*}{ Models } & \multicolumn{2}{c}{ Napier scenario } & MAE & \multicolumn{2}{c}{ Ruzi scenario } \\
& MAE & RMSE & $R^{2}$ & 29.124 & 32.523 \\
\hline GUT-R & 20.122 & 21.634 & 0.267 & 2.112 & 4.682 \\
SVR & 2.125 & 4.782 & 0.933 & 2.016 & 0.121 \\
ANN & 2.025 & 4.439 & 0.950 & 4.407 & 0.935 \\
\hline
\end{tabular}

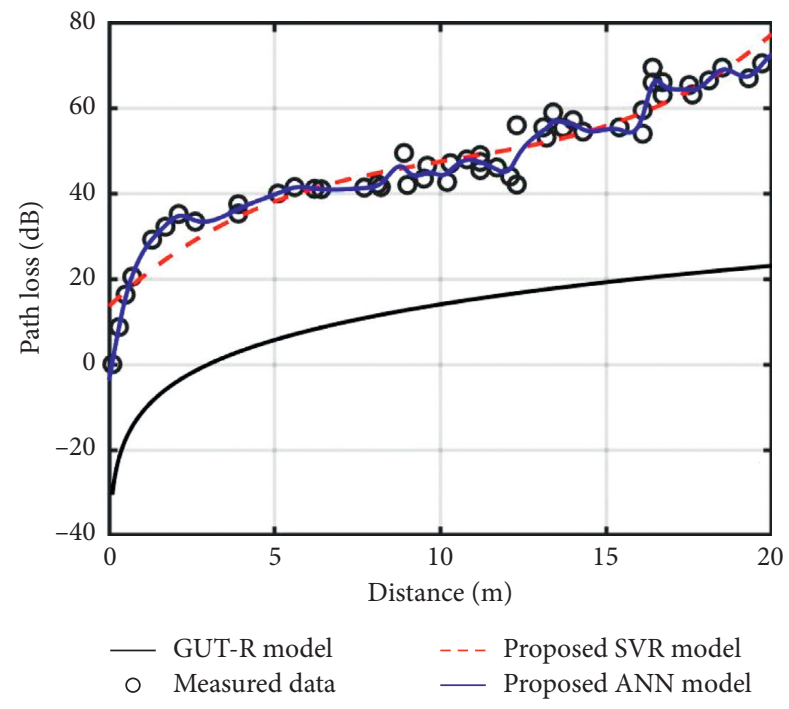

Figure 15: Path loss between UAV trajectory at $5 \mathrm{~m}$ altitudes and GS (Ruzi13) in the Ruzi scenario.

of neurons were four and one output layer, were given a proper prediction model in this work. The hyperplane of RBF-NN can control the iteration of weighting between the training and test sets as easily, which is optimally weighted as 200 iterations. Thus, the $R^{2}$ of the ANN model is 0.967 , or the performance percentage is $97 \%$.
In this work, the performance of the ANN model can smoothly predict the accuracy path loss characteristics better than the SVR model, for example, SF scenarios. Furthermore, we compared the performance of the $R^{2}$ value with the previous works for the path loss prediction models in SF environments, in $[8,30,31]$, as shown in Table 6. 
TABLE 6: Comparison of the $R^{2}$ of the current study with those of previous works.

\begin{tabular}{lccc}
\hline Ref./year & Path loss model & Wireless link & SF scenarios \\
\hline Jawad et al. [8] & EXP-PSO and Poly-PSO & $2.4 \mathrm{GHz}$ Zigbee & Farm field \\
Abouzar et al. [30] & LNSM & $2.4 \mathrm{GHz}$ Zigbee & Apple orchards \\
Raheemah et al. [31] & New LRL method & $2.4 \mathrm{GHz}$ IEEE 802.15.4 & Greenhouse for mangoes \\
This work & SVR & $2.4 \mathrm{GHz}$ Wi-Fi & Napier and Ruzi grass farms \\
This work & ANN & $2.4 \mathrm{GHz}$ Wi-Fi & Napier and Ruzi grass farms \\
\hline
\end{tabular}

EXP-PSO: exponential particle swarm optimization; POLY-PSO: polynomial PSO; LNSM: log-normal shadowing model; and LRL: linear regression line.

\section{Conclusions}

In this paper, the path loss characteristics in realistic propagation SF scenarios for GS-to-UAV-enabled communication were studied by deploying ML models as the prediction models. The measurement data such as RSSI and path loss characteristics are experimentally investigated in different measurement locations such as Napier and Ruzi farm SF scenarios. To predict the accuracy model and build the validation models, the measured data of RSSI and path loss data sets are separated as the training and test sets for the supervised ML models. The performance results show that the supervised method ML models based on SVR and ANN can accurately predict the path loss characterization in SF. It can show that the accuracy is $95 \%$ for the SVR model and $97 \%$ for the ANN model. The performance of the SVR model is less than the ANN model at least $2 \%$ for this work. Furthermore, we show that the ML-based prediction models are more accurate than the empirical GUT-R model. This work is useful for seeking an accurate and reliable channel model for the network planning of GS and UAV communications in SF scenarios.

\section{Data Availability}

The data used to support the findings of this study are available from the corresponding author upon request.

\section{Conflicts of Interest}

The authors declare that there are no conflicts of interest concerning the publication of this paper.

\section{Acknowledgments}

This work was financially supported by the KMITL research fund and TSRI in the Fundamental Fund (FF) scholarship. Furthermore, the authors would like to thank the Tropical Animal Research Institute, Ramkhamhaeng University, Thailand.

\section{References}

[1] J. Kim, S. Kim, C. Ju, and H. I. Son, “Unmanned aerial vehicles in agriculture: a review of perspective of platform, control, and applications," IEEE Access, vol. 7, pp. 105100-105115, 2019.

[2] C. Potena, R. Khanna, J. Nieto, R. Siegwart, D. Nardi, and A. Pretto, "AgriColMap: aerial-ground collaborative 3D mapping for precision farming," IEEE Robotics and Automation Letters, vol. 4, no. 2, pp. 1085-1092, 2019.
[3] W. Bouachir, K. E. Ihou, H.-E. Gueziri, N. Bouguila, and N. Belanger, "Computer vision system for automatic counting of planting microsites using UAV imagery," IEEE Access, vol. 7, pp. 82491-82500, 2019.

[4] C. D. Lopez and L. F. Giraldo, "Optimization of energy and water consumption on crop irrigation using UAVs via path design," in Proceeding of IEEE Colombian Conference on Automatic Control (CCAC), pp. 1-5, Medellin, Colombia, October 2019.

[5] M. Rizk, A. Mroue, M. Farran, and J. Charara, "Real-time SLAM based on image stitching for autonomous navigation of UAVs in GNSS-denied regions," in Proceeding of IEEE International Conference on Artificial Intelligent Circuits and Systems (AICAS), pp. 301-304, Genova, Italy, April 2020.

[6] M. Idbella, M. Iadaresta, G. Gagliarde et al., "A new wireless sensor for monitoring agrometeorological data in areas lacking communication networks," Sensors MDPI, vol. 20, no. 1589, pp. 1-13, 2020.

[7] M. Bacco, A. Berton, A. Gotta, and L. Caviglione, "IEEE 802.15.4 air-ground UAV communications in smart farming scenarios," IEEE Communications Letters, vol. 22, no. 9, pp. 1910-1913, 2018.

[8] H. M. Jawad, A. M. Jawad, R. Nordin et al., "Accurate empirical path-loss model based on particle swarm optimization for wireless sensor networks in smart agriculture," IEEE Sensors Journal, vol. 20, no. 1, pp. 552-561, 2020.

[9] M. Najafzadeh and G. Oliveto, "Riprap incipient motion for overtopping flows with machine learning models," Journal of Hydroinformatics, vol. 22, no. 4, pp. 749-767, 2020.

[10] M. Najafzadeh and A. Ghaemi, "Prediction of the five-day biochemical oxygen demand and chemical oxygen demand in natural streams using machine learning methods," Environmental Monitoring and Assessment, vol. 191, no. 6, pp. 380-21, 2019.

[11] M. Najafzadeh and M. Zeinolabedini, "Prognostication of waste water treatment plant performance using efficient soft computing models: an environmental evaluation," Measurement, vol. 138, pp. 690-701, 2019.

[12] M. Najafzadeh, A. Etemad-Shahidi, and S. Y. Lim, "Scour prediction in long contractions using ANFIS and SVM," Ocean Engineering, vol. 111, pp. 128-135, 2016.

[13] M. Najafzadeh, F. Saberi-Movahed, and S. Sarkamaryan, "NFGMDH-Based self-organized systems to predict bridge pier scour depth under debris flow effects," Marine Georesources \& Geotechnology, vol. 36, no. 5, pp. 589-602, 2018.

[14] M. Najafzadeh and F. Saberi-Movahed, "GMDH-GEP to predict free span expansion rates below pipelines under waves," Marine Georesources \& Geotechnology, vol. 37, no. 3, pp. 375-392, 2019.

[15] F. S-Movahed, M. Najafzadeh, and A. Mehrpooya, "Receiving more accurate predictions for longitudinal dispersion coeffcients in water pipelines: training group method of data 
handling using extreme learning machine conceptions," Water Resources Management, vol. 34, pp. 529-561, 2020.

[16] H. Singh, S. Gupta, C. Dhawan, and A. Misshra, "Path loss prediction in smart campus environment: machine learningbased applications," in Proceeding of IEEE Vehicular Technology Conference (VTC2020-Spring), pp. 1-5, Antwerp, Belgium, May 2020.

[17] S. I. Popoola, A. Jefia, A. A. Atayero et al., "Determination of neural network parameters for path loss prediction in very high frequency wireless channel," IEEE Access, vol. 7, pp. 150462-150483, 2019.

[18] M. Ayadi, A. Ben Zineb, and S. Tabbane, "A UHF path loss model using learning machine for heterogeneous networks," IEEE Transactions on Antennas and Propagation, vol. 65, no. 7, pp. 3675-3683, 2017.

[19] J. Wan, Y. Zhang, G. Yang, Z. He, and W. Zhang, "Path loss prediction based on machine learning methods for aircraft cabin environments," IEEE Access, vol. 7, pp. 159251-159261, 2019.

[20] K.-P. Lin, K.-C. Hung, J.-C. Lin, C.-K. Wang, and P.-F. Pai, "Applying least squares support vector regression with genetic algorithms for radio-wave path-loss prediction in suburban environment," in Advances in Neural Network Research and Applications of Lecture Notes in Electrical Engineering, vol. 67, pp. 861-868, Springer, Berlin, Germany, 2010.

[21] D. Nikitopoulos, P. Constantinou, and I. Nafornita, "ANN prediction models for outdoor environment," in Proceedings of the 2006 IEEE 17th International Symposium on Personal, Indoor and Mobile Radio Communications, pp. 1-5, Helsinki, Finland, September 2006.

[22] J. Tharane, D. Zibar, and H. L. Christiansen, "Model-aided deep learning methods for path loss prediction in mobile communication systems at $2.6 \mathrm{GHz}$," IEEE Access, vol. 8, pp. 7925-7936, 2020.

[23] S. Aldossari and K.-C. Chen, "Predicting the path loss of wireless channel models using machine learning techniques in MmWave urban communications," in Proceeding of the International Symposium on Wireless Personal Multimedia Communications (WPMC), pp. 1-6, Lisbon, Portugal, November 2019.

[24] O. Ahmadian, H. F. Ates, T. Baykas, and B. K. Gunturk, "Prediction path loss distribution of an area from satellite image using deep learning," IEEE Access, vol. 8, pp. 6498264991, 2020.

[25] P. S. Bithas, E. T. Michailidis, N. Nomikos, D. Vouyioukas, and A. G. Kanatas, "A survey on machine-learning techniques for UAV-based communications," Sensors (Basel, Switzerland), vol. 19, no. 5170, pp. 1-39, 2019.

[26] S. Duangsuwan, C. Teekapakvisit, and M. M. Maw, "Development of soil moisture monitoring by using IoT and UAVSC for smart farming application," Advances in Science, Technology and Engineering Systems Journal, vol. 5, no. 4, pp. 381-387, 2020.

[27] Y. Zhang, J. Wen, G. Yang, Z. He, and J. Wang, "Path loss prediction based on machine learning: principle, method, and data expansion," Applied Sciences, vol. 9, no. 9, p. 1908, 2019.

[28] Y. Zhang, J. Wen, G. Yang, Z. He, and X. Luo, "Air-to-air path loss prediction based on machine learning methods in urban environments," Wireless Communications and Mobile Computing, vol. 2018, pp. 1-9, 2018.

[29] L. Shan, R. Miura, T. Kagawa, F. Ono, H.-B. Li, and F. Kojima, "Machine learning-based field data analysis and modeling for drone communications," IEEE Access, vol. 7, pp. 7912779135, 2019.
[30] P. Abouzar, D. G. Michelson, and M. Hamdi, "RSSI-based distributed self-localization for wireless sensor networks used in precision agriculture," IEEE Transactions on Wireless Communications, vol. 15, no. 10, pp. 6638-6650, 2016.

[31] A. Raheemah, N. Sabri, M. S. Salim, P. Ehkan, and R. B. Ahmad, "New empirical path loss model for wireless sensor networks in mango greenhouses," Computers and Electronics in Agriculture, vol. 127, pp. 553-560, 2016. 